PROCEEDINGS OF THE

AMERICAN MATHEMATICAL SOCIETY

Volume 138, Number 12, December 2010, Pages 4291-4302

S 0002-9939(2010)10510-5

Article electronically published on May 26, 2010

\title{
WEIGHTED ORLICZ-RIESZ CAPACITY OF BALLS
}

\author{
YOSHIHIRO MIZUTA, TAKAO OHNO, AND TETSU SHIMOMURA \\ (Communicated by Tatiana Toro)
}

\begin{abstract}
Our aim in this paper is to estimate the weighted Orlicz-Riesz capacity of balls.
\end{abstract}

\section{Introduction AND STATEMENT OF RESUlts}

Several versions of capacities for Orlicz-Riesz spaces have appeared in research papers, for example those by Aissaoui and Benkirane [5], Kuznetsov 14, Mizuta [17, Adams and Hurri-Syrjänen [3, 4], and Joensuu [12. The notion of capacity offers a standard way to characterize exceptional sets and is indispensable to an understanding of the local behavior of functions in Orlicz-Riesz spaces. Various capacity estimates also play an important role in the study of solutions to partial differential equations.

Recently the authors 8, gave an estimate of the Orlicz-Riesz capacity of balls as an extension of Adams and Hurri-Syrjänen [4] and Joensuu [12]. An estimate of the weighted Sobolev capacity of balls can be found e.g. in Heinonen, Kilpeläinen and Martio 9 .

A positive measurable function $w$ on $\mathbf{R}^{n}$ is called an $A_{p}$ weight (written as $\left.w \in A_{p}\right)$ if there exists a positive constant $C_{p}$ such that

$$
\left(\frac{w(B)}{|B|}\right)\left(\frac{w^{1 /(1-p)}(B)}{|B|}\right)^{p-1} \leq C_{p} \quad(<\infty)
$$

for all balls $B$, where $1<p<\infty,|\cdot|$ denotes the $n$-dimensional Lebesgue measure and

$$
w(B)=\int_{B} w(y) d y .
$$

As an example, we have that the function $w(x)=|x|^{\delta}$ is an $A_{p}$ weight if and only if $-n<\delta<n(p-1)$. It is well known that, for an $A_{p}$ weight $w$, the corresponding measure $w$ is doubling, that is, $w(2 B) \leq c w(B)$ for all balls $B=B(x, r)$; here the constant $c$ depends only on $n, p$ and $C_{p}$ and $2 B$ stands for the enlarged ball $B(x, 2 r)$. For these and other fundamental properties of $A_{p}$ weights, see, for example, Heinonen, Kilpeläinen and Martio [9].

Received by the editors January 25, 2010.

2010 Mathematics Subject Classification. Primary 46E35; Secondary 46E30, 31 B15.

Key words and phrases. Weighted Orlicz-Riesz capacity, Riesz potential, weighted-Riesz spaces.

(C)2010 American Mathematical Society 
For $0<\alpha<n$ and a locally integrable function $f$ on $\mathbf{R}^{n}$, we define the Riesz potential $I_{\alpha} f$ of order $\alpha$ by

$$
I_{\alpha} f(x)=\int_{\mathbf{R}^{n}}|x-y|^{\alpha-n} f(y) d y .
$$

In the present paper, we treat functions $f$ satisfying an Orlicz condition with an $A_{p}$ weight $\omega$ :

$$
\int_{\mathbf{R}^{n}} \varphi_{p}(|f(y)|) \omega(y) d y<\infty .
$$

Here $\varphi_{p}(r)$ is a positive nondecreasing function on the interval $(0, \infty)$ of the form

$$
\varphi_{p}(r)=r^{p} \varphi(r)
$$

where $p>1$ and $\varphi(r)$ is a positive quasi-increasing function on $(0, \infty)$ which is of logarithmic type; that is, there exists $c_{1}>0$ such that

$$
c_{1}^{-1} \varphi(r) \leq \varphi\left(r^{2}\right) \leq c_{1} \varphi(r) \quad \text { whenever } r>0 .
$$

We say that $\varphi(r)$ is quasi-increasing if there exists $c>0$ such that

$$
\varphi(s) \leq c \varphi(t) \quad \text { whenever } 0<s<t .
$$

We set

$$
\varphi_{p}(0)=0,
$$

because we will see from $(\varphi 4)$ below that

$$
\lim _{r \rightarrow 0+} \varphi_{p}(r)=0
$$

see [19, p. 205].

For an open set $G \subset \mathbf{R}^{n}$, we denote by $L^{\varphi_{p}, \omega}(G)$ the family of all locally integrable functions $f$ on $G$ such that

$$
\int_{G} \varphi_{p}(|f(y)|) \omega(y) d y<\infty
$$

and define

$$
\|f\|_{\varphi_{p}, \omega, G}=\inf \left\{\lambda>0: \int_{G} \varphi_{p}(|f(y)| / \lambda) \omega(y) d y \leq 1\right\} .
$$

This is a quasi-norm in $L^{\varphi_{p}, \omega}(G)$. For $E \subset G$, the relative $\left(\alpha, \varphi_{p}, \omega\right)$-capacity is defined by

$$
B_{\alpha, \varphi_{p}, \omega}(E ; G)=\inf \int_{G} \varphi_{p}(|f(y)|) \omega(y) d y,
$$

where the infimum is taken over all functions $f$ such that $f=0$ outside $G$ and

$$
I_{\alpha} f(x) \geq 1 \quad \text { for all } x \in E
$$

(cf. Adams and Hedberg [2], Meyers [15, Ziemer [22] and the first author [16, 17]).

Our first aim in the present note is to give an estimate of the modular capacity $B_{\alpha, \varphi_{p}, \omega}$ of open balls $B(x, r)$ centered at $x$ of radius $r$, as an extension of Adams and Hurri-Syrjänen [4, Theorem 2.11], Joensuu [12, Heinonen, Kilpeläinen and Martio [9, Theorems 2.18 and 2.19] and the authors [8, Theorem A]. In fact, our first theorem is stated in the following. 
Theorem A (cf. [16, Lemma 7.3]). Suppose $p>1$ and $\omega \in A_{p}$. For $R>0$, there exists a constant $A>0$ such that

$$
\begin{aligned}
& A^{-1}\left(\int_{r}^{R}\left\{t^{-\alpha p} \varphi\left(t^{-1}\right) \omega(B(x, t))\right\}^{1 /(1-p)} d t / t\right)^{1-p} \leq B_{\alpha, \varphi_{p}, \omega}(B(x, r) ; B(0, R)) \\
& \quad \leq A\left(\int_{r}^{R}\left\{t^{-\alpha p} \varphi\left(t^{-1}\right) \omega(B(x, t))\right\}^{1 /(1-p)} d t / t\right)^{1-p}
\end{aligned}
$$

whenever $B(x, r) \subset B(0, R / 4)$.

We write $f \sim g$ if there exists a constant $A$ so that $A^{-1} g \leq f \leq A g$.

Example 1.1. Let $\omega(x)=|x|^{\delta}$ and $\varphi(t)=(\log (e+t))^{\beta}$.

(1) If $\alpha p-n<\delta<n(p-1)$, then

$\int_{r}^{R}\left\{t^{-\alpha p} \varphi\left(t^{-1}\right) \omega(B(0, t))\right\}^{1 /(1-p)} d t / t \sim r^{(-\alpha p+n+\delta) /(1-p)}(\log (e+1 / r))^{\beta /(1-p)}$

for $0<r<R / 2<1$. In this case,

$$
B_{\alpha, \varphi_{p}, \omega}(B(0, r) ; B(0, R)) \sim r^{-\alpha p+n+\delta}(\log (e+1 / r))^{\beta} .
$$

(2) If $\alpha p-n=\delta$ and $\beta<p-1$, then

$$
\int_{r}^{R}\left\{t^{-\alpha p} \varphi\left(t^{-1}\right) \omega(B(0, t))\right\}^{1 /(1-p)} d t / t \sim(\log (e+1 / r))^{\beta /(1-p)+1}
$$

for $0<r<R / 2<1$. In this case,

$$
B_{\alpha, \varphi_{p}, \omega}(B(0, r) ; B(0, R)) \sim(\log (e+1 / r))^{\beta+1-p} .
$$

(3) If $\alpha p-n=\delta$ and $\beta=p-1$, then

$$
\int_{r}^{R}\left\{t^{-\alpha p} \varphi\left(t^{-1}\right) \omega(B(0, t))\right\}^{1 /(1-p)} d t / t \sim \log (e+(\log (e+1 / r)))
$$

for $0<r<R / 2<1$. In this case,

$$
B_{\alpha, \varphi_{p}, \omega}(B(0, r) ; B(0, R)) \sim(\log (e+(\log (e+1 / r))))^{-(p-1)} .
$$

(4) If $\alpha p-n>\delta>-n$ or $\alpha p-n=\delta$ and $\beta>p-1$, then

$$
B_{\alpha, \varphi_{p}, \omega}(\{0\} ; B(0,1))>0 .
$$

Next we are concerned with the norm capacity. For $E \subset G$, we define

$$
C_{\alpha, \varphi_{p}, \omega}(E ; G)=\inf \|f\|_{\varphi_{p}, \omega, G},
$$

where the infimum is taken over all functions $f$ such that $f=0$ outside $G$ and

$$
I_{\alpha} f(x) \geq 1 \quad \text { for all } x \in E .
$$


Theorem B. Suppose $p>1$ and $\omega \in A_{p}$. For $R>0$, there exists a constant $A>0$ such that

$$
\begin{aligned}
& A^{-1}\left(\int_{r}^{R}\left\{t^{-\alpha p} \varphi\left(t^{-1}\right) \omega(B(x, t))\right\}^{1 /(1-p)} d t / t\right)^{(1-p) / p} \leq C_{\alpha, \varphi_{p}, \omega}(B(x, r) ; B(0, R)) \\
& \quad \leq A\left(\int_{r}^{R}\left\{t^{-\alpha p} \varphi\left(t^{-1}\right) \omega(B(x, t))\right\}^{1 /(1-p)} d t / t\right)^{(1-p) / p}
\end{aligned}
$$

whenever $B(x, r) \subset B(0, R / 4)$.

In view of Theorems $\mathrm{A}$ and $\mathrm{B}$, we have the following result, which extends the results by Adams and Hurri-Syrjänen [4, Joensuu [12] and the authors [8].

Corollary 1.2. Suppose $p>1$ and $\omega \in A_{p}$. For $R>0$, there is a constant $A>0$ such that

$$
\begin{aligned}
A^{-1} B_{\alpha, \varphi_{p}, \omega} & (B(x, r) ; B(0, R))^{1 / p} \\
\leq & C_{\alpha, \varphi_{p}, \omega}(B(x, r) ; B(0, R)) \leq A B_{\alpha, \varphi_{p}, \omega}(B(x, r) ; B(0, R))^{1 / p}
\end{aligned}
$$

whenever $B(x, r) \subset B(0, R / 4)$.

For further related results, we refer the reader to Adams [1], Adams and HurriSyrjänen [3, Edmunds and Evans [7], Kilpeläinen [13] and Mizuta and Shimomura [19, 20, 21].

Throughout this paper, let $A$ denote various constants independent of the variables in question and let $A(a, b, \cdots)$ be a constant that depends on $a, b, \cdots$.

\section{Proof of Theorem A}

First we collect properties which follow from condition $(\varphi 1)$ (see [17, [19, Lemma 2.3], [18, Section 7]).

$(\varphi 2) \varphi$ satisfies the doubling condition; that is, there exists $c_{2}>1$ such that

$$
c_{2}^{-1} \varphi(r) \leq \varphi(2 r) \leq c_{2} \varphi(r) \quad \text { whenever } r>0 .
$$

$(\varphi 3)$ For each $\gamma>0$, there exists $c_{3}=c_{3}(\gamma) \geq 1$ such that

$$
c_{3}^{-1} \varphi(r) \leq \varphi\left(r^{\gamma}\right) \leq c_{3} \varphi(r) \quad \text { whenever } r>0 .
$$

$(\varphi 4)$ For each $\gamma>0$, there exists $c_{4}=c_{4}(\gamma) \geq 1$ such that

$$
s^{\gamma} \varphi(s) \leq c_{4} t^{\gamma} \varphi(t) \quad \text { whenever } 0<s<t .
$$

$(\varphi 5)$ For each $\gamma>0$, there exists $c_{5}=c_{5}(\gamma) \geq 1$ such that

$$
t^{-\gamma} \varphi(t) \leq c_{5} s^{-\gamma} \varphi(s) \quad \text { whenever } 0<s<t .
$$

$(\varphi 6)$ If $\varphi$ and $\psi$ are positive monotone functions on $[0, \infty)$ satisfying $(\varphi 1)$, then for each $\gamma>0$, there exists a constant $c_{6}=c_{6}(\gamma) \geq 1$ such that

$$
c_{6}{ }^{-1} \varphi(r) \leq \varphi\left(r^{\gamma} \psi(r)\right) \leq c_{6} \varphi(r) \quad \text { whenever } r>0 .
$$

Let us begin with an upper estimate for the modular $B_{\alpha, \varphi_{p}, \omega}$-capacity of balls. 
Theorem 2.1. Suppose $p>1$ and $\omega$ is a positive locally integrable function on $\mathbf{R}^{n}$. Then there exists a constant $A>0$ such that

$$
B_{\alpha, \varphi_{p}, \omega}(B(0, r) ; B(0, R)) \leq A\left(\int_{2 r}^{2 R}\left\{t^{-\alpha p} \varphi\left(t^{-1}\right) \omega(B(0, t))\right\}^{1 /(1-p)} d t / t\right)^{1-p}
$$

whenever $0<r<R / 2<\infty$.

Proof. For $r>0$, consider the function

$$
f_{r}(y)=|y|^{-\alpha}
$$

for $r<|y|<2 r$ and $f_{r}=0$ elsewhere. If $x \in B(0, r)$ and $y \in B(0,2 r) \backslash B(0, r)$, then $|x-y|<3 r$, so that

$$
I_{\alpha} f_{r}(x) \geq(3 r)^{\alpha-n} \int_{B(0,2 r) \backslash B(0, r)}|y|^{-\alpha} d y=A_{1}
$$

with a constant $A_{1}=A_{1}(\alpha, n)>0$.

Now let $0<r<R / 2$, and take $j_{0}$ such that $2^{j_{0}+1} r \leq R<2^{j_{0}+2} r$. For $\left\{a_{j}\right\}$ such that $a_{j} \geq 0$ and $\sum_{j=0}^{j_{0}} a_{j}=1$, set

$$
f=\sum_{j=0}^{j_{0}} a_{j} f_{2^{j} r} / A_{1}
$$

Then

$$
I_{\alpha} f(x)=A_{1}^{-1} \sum_{j=0}^{j_{0}} a_{j} I_{\alpha} f_{2^{j} r}(x) \geq 1
$$

for $x \in B(0, r)$. Therefore we have by $(\varphi 2)$ and $(\varphi 3)$ that

$$
\begin{aligned}
B_{\alpha, \varphi_{p}, \omega}(B(0, r) ; B(0, R)) & \leq \int_{B(0, R)} \varphi_{p}(f(y)) \omega(y) d y \\
& =\sum_{j=0}^{j_{0}} \int_{B(0, R)}\left[a_{j} f_{2^{j} r}(y) / A_{1}\right]^{p} \varphi\left(a_{j} f_{2^{j} r}(y) / A_{1}\right) \omega(y) d y \\
& \leq A_{2} \sum_{j=0}^{j_{0}} a_{j}^{p}\left(2^{j} r\right)^{-\alpha p} \varphi\left(\left(2^{j} r\right)^{-1}\right) \omega\left(B\left(0,2^{j+1} r\right)\right) .
\end{aligned}
$$

Now, letting $K=\sum_{j=0}^{j_{0}}\left\{\left(2^{j} r\right)^{-\alpha p} \varphi\left(\left(2^{j} r\right)^{-1}\right) \omega\left(B\left(0,2^{j+1} r\right)\right)\right\}^{1 /(1-p)}$ and

$$
a_{j}=\frac{\left\{\left(2^{j} r\right)^{-\alpha p} \varphi\left(\left(2^{j} r\right)^{-1}\right) \omega\left(B\left(0,2^{j+1} r\right)\right)\right\}^{1 /(1-p)}}{K},
$$

we find

$$
\begin{aligned}
B_{\alpha, \varphi_{p}, \omega}(B(0, r) ; B(0, R)) & \leq A_{2} K^{-p} \sum_{j=0}^{j_{0}}\left\{\left(2^{j} r\right)^{-\alpha p} \varphi\left(\left(2^{j} r\right)^{-1}\right) \omega\left(B\left(0,2^{j+1} r\right)\right)\right\}^{1 /(1-p)} \\
& =A_{2} K^{1-p}
\end{aligned}
$$


Here we have

$$
\begin{aligned}
K & \geq A_{3} \sum_{j=0}^{j_{0}} \int_{2^{j+1} r}^{2^{j+3} r}\left\{t^{-\alpha p} \varphi\left(t^{-1}\right) \omega(B(0, t))\right\}^{1 /(1-p)} d t / t \\
& \geq A_{3} \int_{2 r}^{2^{j 0+3} r}\left\{t^{-\alpha p} \varphi\left(t^{-1}\right) \omega(B(0, t))\right\}^{1 /(1-p)} d t / t,
\end{aligned}
$$

so that

$$
K \geq A_{3} \int_{2 r}^{2 R}\left\{t^{-\alpha p} \varphi\left(t^{-1}\right) \omega(B(0, t))\right\}^{1 /(1-p)} d t / t,
$$

which proves the result.

Corollary 2.2. If $\omega$ is doubling, then there exists a constant $A>0$ such that

$$
B_{\alpha, \varphi_{p}, \omega}(B(x, r) ; B(0, R)) \leq A\left(\int_{r}^{R}\left\{t^{-\alpha p} \varphi\left(t^{-1}\right) \omega(B(x, t))\right\}^{1 /(1-p)} d t / t\right)^{1-p}
$$

whenever $B(x, r) \subset B(0, R / 4)$. In fact,

$$
\begin{aligned}
B_{\alpha, \varphi_{p}, \omega}(B(x, r) ; B(0, R)) & \leq B_{\alpha, \varphi_{p}, \omega}(B(x, r) ; B(x, R / 2)) \\
& \leq A\left(\int_{r}^{R}\left\{t^{-\alpha p} \varphi\left(t^{-1}\right) \omega(B(x, t))\right\}^{1 /(1-p)} d t / t\right)^{1-p}
\end{aligned}
$$

whenever $B(x, r) \subset B(0, R / 4)$.

Next we give a lower estimate for the modular $B_{\alpha, \varphi_{p}, \omega}$-capacity of balls.

Theorem 2.3. Suppose $p>1$ and $\omega \in A_{p}$. For $R>0$, there exists a constant $A=A(R)>0$ such that

$$
B_{\alpha, \varphi_{p}, \omega}(B(0, r) ; B(0, R)) \geq A\left(\int_{r}^{R}\left\{t^{-\alpha p} \varphi\left(t^{-1}\right) \omega(B(0, t))\right\}^{1 /(1-p)} d t / t\right)^{1-p}
$$

whenever $0<r<R / 2<\infty$.

Proof. For $0<r<R / 2$, take a nonnegative measurable function $f$ on $B(0, R)$ such that

$$
I_{\alpha} f(x) \geq 1 \quad \text { for } x \in B(0, r) .
$$

Then we have by Fubini's theorem

$$
\begin{aligned}
\int_{B(0, r)} d x & \leq \int_{B(0, r)} I_{\alpha} f(x) d x \\
& \leq \int_{B(0, R)}\left(\int_{B(0, r)}|x-y|^{\alpha-n} d x\right) f(y) d y \\
& \leq A_{1} r^{n} \int_{B(0, R)}(r+|y|)^{\alpha-n} f(y) d y .
\end{aligned}
$$


For $\varepsilon>0$ and $0<\delta<\alpha$, we see from Hölder's inequality that

$$
\begin{aligned}
& \int_{B(0, R)}(r+|y|)^{\alpha-n} f(y) d y \\
= & \int_{\left\{y \in B(0, R): f(y)>\varepsilon(r+|y|)^{-\delta}\right\}}(r+|y|)^{\alpha-n} f(y) d y \\
& +\int_{\left\{y \in B(0, R): f(y) \leq \varepsilon(r+|y|)^{-\delta}\right\}}(r+|y|)^{\alpha-n} f(y) d y \\
\leq & \left(\int_{B(0, R)}\left[(r+|y|)^{\alpha-n}\left\{\varphi\left(\varepsilon(r+|y|)^{-\delta}\right) \omega(y)\right\}^{-1 / p}\right]^{p^{\prime}} d y\right)^{1 / p^{\prime}} \\
& \times\left(\int_{B(0, R)} \varphi_{p}(f(y)) \omega(y) d y\right)^{1 / p}+\varepsilon \int_{B(0, R)}(r+|y|)^{\alpha-n-\delta} d y,
\end{aligned}
$$

where $1 / p+1 / p^{\prime}=1$. Since $\omega \in A_{p}$, we find by $(\varphi 2)$ and $(\varphi 3)$ that

$$
\begin{aligned}
& \int_{B(0, R)}\left[(r+|y|)^{\alpha-n}\left\{\varphi\left(\varepsilon(r+|y|)^{-\delta}\right) \omega(y)\right\}^{-1 / p}\right]^{p^{\prime}} d y \\
\leq & A_{2}(\varepsilon) \int_{0}^{R}(r+t)^{(\alpha-n) p /(p-1)} \varphi\left((r+t)^{-1}\right)^{1 /(1-p)}\left(\int_{B(0, t)} \omega(y)^{1 /(1-p)} d y\right) \frac{d t}{t} \\
\leq & A_{3}(\varepsilon) \int_{0}^{R}(r+t)^{\alpha p /(p-1)} \varphi\left((r+t)^{-1}\right)^{1 /(1-p)}\left(\int_{B(0, r+t)} \omega(y) d y\right)^{1 /(1-p)} \frac{d t}{t} \\
\leq & A_{3}(\varepsilon) \int_{r}^{2 R}\left\{t^{-\alpha p} \varphi\left(t^{-1}\right) \omega(B(0, t))\right\}^{1 /(1-p)} d t / t \\
\leq & A_{4}(\varepsilon) \int_{r}^{R}\left\{t^{-\alpha p} \varphi\left(t^{-1}\right) \omega(B(0, t))\right\}^{1 /(1-p)} d t / t .
\end{aligned}
$$

Thus we derive

$$
\begin{aligned}
& \int_{B(0, R)}(r+|y|)^{\alpha-n} f(y) d y \\
\leq & A_{4}(\varepsilon)\left(\int_{r}^{R}\left\{t^{-\alpha p} \varphi\left(t^{-1}\right) \omega(B(0, t))\right\}^{1 /(1-p)} d t / t\right)^{1 / p^{\prime}} \\
& \times\left(\int_{B(0, R)} \varphi_{p}(f(y)) \omega(y) d y\right)^{1 / p}+A_{5} \varepsilon
\end{aligned}
$$

so that

$$
\begin{aligned}
1 \leq & A_{6}(\varepsilon)\left(\int_{r}^{R}\left\{t^{-\alpha p} \varphi\left(t^{-1}\right) \omega(B(0, t))\right\}^{1 /(1-p)} d t / t\right)^{1 / p^{\prime}} \\
& \times\left(\int_{B(0, R)} \varphi_{p}(f(y)) \omega(y) d y\right)^{1 / p}+A_{7} \varepsilon
\end{aligned}
$$


If $A_{7} \varepsilon=1 / 2$, then we establish

$$
B_{\alpha, \varphi_{p}, \omega}(B(0, r) ; B(0, R)) \geq A\left(\int_{r}^{R}\left\{t^{-\alpha p} \varphi\left(t^{-1}\right) \omega(B(0, t))\right\}^{1 /(1-p)} d t / t\right)^{1-p},
$$

as required.

Corollary 2.4. Suppose $p>1$ and $\omega \in A_{p}$. For $R>0$, there exists a constant $A=A(R)>0$ such that

$$
B_{\alpha, \varphi_{p}, \omega}(B(x, r) ; B(0, R)) \geq A\left(\int_{r}^{R}\left\{t^{-\alpha p} \varphi\left(t^{-1}\right) \omega(B(x, t))\right\}^{1 /(1-p)} d t / t\right)^{1-p}
$$

whenever $B(x, r) \subset B(0, R / 4)$.

Now Theorem A follows from Corollaries 2.2 and 2.4 .

\section{Proof of Theorem B}

Let us begin with an upper estimate for the norm $C_{\alpha, \varphi_{p}, \omega}$-capacity of balls.

Theorem 3.1. Suppose that $p>1$ and that $\omega(B(0, r))$ satisfies the doubling condition. Then there exists a constant $A>0$ such that

$$
C_{\alpha, \varphi_{p}, \omega}(B(0, r) ; B(0, R)) \leq A\left(\int_{r}^{R}\left\{t^{-\alpha p} \varphi\left(t^{-1}\right) \omega(B(0, t))\right\}^{1 /(1-p)} d t / t\right)^{(1-p) / p}
$$

whenever $0<r<R / 2<\infty$.

Proof. Set

$$
\varphi^{*}(r)=\int_{r}^{R}\left\{t^{-\alpha p} \varphi\left(t^{-1}\right) \omega(B(0, t))\right\}^{1 /(1-p)} d t / t
$$

for $r>0$. Consider the function

$$
f(y)=|y|^{-\alpha}\left\{|y|^{-\alpha p} \varphi\left(|y|^{-1}\right) \omega(B(0,|y|))\right\}^{1 /(1-p)} \varphi^{*}(r)^{-1 / p}
$$

for $r<|y|<R$ and $f=0$ elsewhere. If $x \in B(0, r)$ and $y \in B(0, R) \backslash B(0, r)$, then $|x-y|<2|y|$, so that

$$
\begin{aligned}
I_{\alpha} f(x) & \geq 2^{\alpha-n} \varphi^{*}(r)^{-1 / p} \int_{B(0, R) \backslash B(0, r)}|y|^{-n}\left\{|y|^{-\alpha p} \varphi\left(|y|^{-1}\right) \omega(B(0,|y|))\right\}^{1 /(1-p)} d y \\
& =A_{1} \varphi^{*}(r)^{(p-1) / p}
\end{aligned}
$$

with a constant $A_{1}=A_{1}(\alpha, n)>0$. It follows from the definition of capacity that

$$
C_{\alpha, \varphi_{p}, \omega}(B(0, r) ; B(0, R)) \leq A_{1}^{-1} \varphi^{*}(r)^{(1-p) / p}\|f\|_{\varphi_{p}, \omega, B(0, R)} .
$$

Thus it suffices to show that

$$
\|f\|_{\varphi_{p}, \omega, B(0, R)} \leq A_{2} .
$$


For this purpose, we first note that

$$
\begin{aligned}
& \int_{B(0, R)} \varphi_{p}(f(y)) \omega(y) d y \\
& =\int_{B(0, R) \backslash B(0, r)} f(y)^{p} \varphi(f(y)) \omega(y) d y \\
& =\varphi^{*}(r)^{-1} \int_{B(0, R) \backslash B(0, r)}|y|^{-\alpha p}\left\{|y|^{-\alpha p} \varphi\left(|y|^{-1}\right) \omega(B(0,|y|))\right\}^{p /(1-p)} \\
& \quad \times \varphi\left(|y|^{-\alpha}\left\{|y|^{-\alpha p} \varphi\left(|y|^{-1}\right) \omega(B(0,|y|))\right\}^{1 /(1-p)} \varphi^{*}(r)^{-1 / p}\right) \omega(y) d y .
\end{aligned}
$$

Here we see from the doubling condition of $\omega(B(0, r))$ that

$$
\begin{gathered}
|y|^{-\alpha}\left\{|y|^{-\alpha p} \varphi\left(|y|^{-1}\right) \omega(B(0,|y|))\right\}^{1 /(1-p)} \varphi^{*}(r)^{-1 / p} \\
\leq A_{3}|y|^{-\alpha}\left\{|y|^{-\alpha p} \varphi\left(|y|^{-1}\right) \omega(B(0,|y|))\right\}^{1 /(1-p)} \\
\left(\int_{r}^{2 R}\left\{t^{-\alpha p} \varphi\left(t^{-1}\right) \omega(B(0, t))\right\}^{1 /(1-p)} d t / t\right)^{-1 / p} \\
\leq A_{3}|y|^{-\alpha}\left\{|y|^{-\alpha p} \varphi\left(|y|^{-1}\right) \omega(B(0,|y|))\right\}^{1 /(1-p)} \\
\left(\int_{|y|}^{2|y|}\left\{t^{-\alpha p} \varphi\left(t^{-1}\right) \omega(B(0, t))\right\}^{1 /(1-p)} d t / t\right)^{-1 / p} \\
\leq A_{4} \varphi\left(|y|^{-1}\right)^{-1 / p} \omega(B(0,|y|))^{-1 / p}
\end{gathered}
$$

for $y \in B(0, R) \backslash B(0, r)$. Further we can find constants $A_{0}>0$ and $\gamma>0$ such that

$$
\omega(B(0, t)) \geq A_{0} t^{\gamma}
$$

for all $t>0$. Hence, as in Theorem 2.1, we obtain by $(\varphi 6)$ :

$$
\begin{aligned}
& \int_{B(0, R)} \varphi_{p}(f(y)) \omega(y) d y \\
& \leq A_{5} \varphi^{*}(r)^{-1} \int_{B(0, R) \backslash B(0, r)}|y|^{-\alpha p}\left\{|y|^{-\alpha p} \varphi\left(|y|^{-1}\right) \omega(B(0,|y|))\right\}^{p /(1-p)} \varphi\left(|y|^{-1}\right) \omega(y) d y \\
& \leq A_{6} \varphi^{*}(r)^{-1} \int_{r}^{R}\left\{t^{-\alpha p} \varphi\left(t^{-1}\right) \omega(B(0, t))\right\}^{1 /(1-p)} d t / t \\
& =A_{6}
\end{aligned}
$$

as required.

Corollary 3.2. Suppose that $p>1$ and $\omega$ is doubling. Then there exists a constant $A>0$ such that

$$
C_{\alpha, \varphi_{p}, \omega}(B(x, r) ; B(0, R)) \leq A\left(\int_{r}^{R}\left\{t^{-\alpha p} \varphi\left(t^{-1}\right) \omega(B(x, t))\right\}^{1 /(1-p)} d t / t\right)^{(1-p) / p}
$$

whenever $B(x, r) \subset B(0, R / 4)$.

Next we give a lower estimate for the norm $C_{\alpha, \varphi_{p}, \omega}$-capacity of balls. 
Theorem 3.3. Suppose $p>1$ and $\omega \in A_{p}$. For $R>0$, there exists a constant $A=A(R)>0$ such that

$$
C_{\alpha, \varphi_{p}, \omega}(B(0, r) ; B(0, R)) \geq A\left(\int_{r}^{R}\left\{t^{-\alpha p} \varphi\left(t^{-1}\right) \omega(B(0, t))\right\}^{1 /(1-p)} d t / t\right)^{(1-p) / p}
$$

whenever $0<r<R / 2<\infty$.

Proof. For $0<r<R / 2$ take a nonnegative measurable function $f$ on $B(0, R)$ such that

$$
I_{\alpha} f(x) \geq 1 \quad \text { for } x \in B(0, r) .
$$

Then we have by Fubini's theorem

$$
\begin{aligned}
\int_{B(0, r)} d x & \leq \int_{B(0, r)} I_{\alpha} f(x) d x \\
& \leq \int_{B(0, R)}\left(\int_{B(0, r)}|x-y|^{\alpha-n} d x\right) f(y) d y \\
& \leq A_{1} r^{n} \int_{B(0, R)}(r+|y|)^{\alpha-n} f(y) d y
\end{aligned}
$$

so that

$$
1 \leq A_{1} \int_{B(0, R)}(r+|y|)^{\alpha-n} f(y) d y .
$$

We show that

$$
\begin{aligned}
\int_{B(0, R)} & (r+|y|)^{\alpha-n} f(y) d y \\
& \leq A_{2}\left(\int_{r}^{R}\left\{t^{-\alpha p} \varphi\left(t^{-1}\right) \omega(B(0, t))\right\}^{1 /(1-p)} d t / t\right)^{(p-1) / p}\|f\|_{\varphi_{p}, \omega, B(0, R)} .
\end{aligned}
$$

For this purpose, suppose that $\|f\|_{\varphi_{p}, \omega, B(0, R)} \leq 1$. For $0<\delta<\alpha$, we see from Hölder's inequality that

$$
\begin{aligned}
& \int_{B(0, R)}(r+|y|)^{\alpha-n} f(y) d y \\
= & \int_{\left\{y \in B(0, R): f(y)>(r+|y|)^{-\delta}\right\}}(r+|y|)^{\alpha-n} f(y) d y \\
& +\int_{\left\{y \in B(0, R): f(y) \leq(r+|y|)^{-\delta}\right\}}(r+|y|)^{\alpha-n} f(y) d y \\
\leq & \left(\int_{B(0, R)}\left[(r+|y|)^{\alpha-n}\left\{\varphi\left((r+|y|)^{-\delta}\right) \omega(y)\right\}^{-1 / p}\right]^{p^{\prime}} d y\right)^{1 / p^{\prime}} \\
& \times\left(\int_{B(0, R)} \varphi_{p}(f(y)) \omega(y) d y\right)^{1 / p}+\int_{B(0, R)}(r+|y|)^{\alpha-n-\delta} d y .
\end{aligned}
$$


Here note from $\omega \in A_{p},(\varphi 2)$ and $(\varphi 3)$ that

$$
\begin{aligned}
& \int_{B(0, R)}\left[(r+|y|)^{\alpha-n}\left\{\varphi\left((r+|y|)^{-\delta}\right) \omega(y)\right\}^{-1 / p}\right]^{p^{\prime}} d y \\
\leq & A_{3} \int_{0}^{R}(r+t)^{(\alpha-n) p /(p-1)} \varphi\left((r+t)^{-1}\right)^{1 /(1-p)}\left(\int_{B(0, t)} \omega(y)^{1 /(1-p)} d y\right) \frac{d t}{t} \\
\leq & A_{4} \int_{0}^{R}(r+t)^{\alpha p /(p-1)} \varphi\left((r+t)^{-1}\right)^{1 /(1-p)}\left(\int_{B(0, r+t)} \omega(y) d y\right)^{1 /(1-p)} \frac{d t}{t} \\
\leq & A_{4} \int_{r}^{2 R}\left\{t^{-\alpha p} \varphi\left(t^{-1}\right) \omega(B(0, t))\right\}^{1 /(1-p)} d t / t \\
\leq & A_{5} \int_{r}^{R}\left\{t^{-\alpha p} \varphi\left(t^{-1}\right) \omega(B(0, t))\right\}^{1 /(1-p)} d t / t
\end{aligned}
$$

so that

$$
\begin{aligned}
& \int_{B(0, R)}(r+|y|)^{\alpha-n} f(y) d y \\
\leq & A_{5}\left(\int_{r}^{R}\left\{t^{-\alpha p} \varphi\left(t^{-1}\right) \omega(B(0, t))\right\}^{1 /(1-p)} d t / t\right)^{1 / p^{\prime}} \\
& \times\left(\int_{B(0, R)} \varphi_{p}(f(y)) \omega(y) d y\right)^{1 / p}+A_{6} \\
\leq & A_{7}\left(\int_{r}^{R}\left\{t^{-\alpha p} \varphi\left(t^{-1}\right) \omega(B(0, t))\right\}^{1 /(1-p)} d t / t\right)^{1 / p^{\prime}} .
\end{aligned}
$$

Hence we establish

$$
C_{\alpha, \varphi_{p}, \omega}(B(0, r) ; B(0, R)) \geq A\left(\int_{r}^{R}\left\{t^{-\alpha p} \varphi\left(t^{-1}\right) \omega(B(0, t))\right\}^{1 /(1-p)} d t / t\right)^{(1-p) / p},
$$

as required.

Corollary 3.4. Suppose $p>1$ and $\omega \in A_{p}$. For $R>0$, there exists a constant $A=A(R)>0$ such that

$$
C_{\alpha, \varphi_{p}, \omega}(B(x, r) ; B(0, R)) \geq A\left(\int_{r}^{R}\left\{t^{-\alpha p} \varphi\left(t^{-1}\right) \omega(B(x, t))\right\}^{1 /(1-p)} d t / t\right)^{(1-p) / p}
$$

whenever $B(x, r) \subset B(0, R / 4)$.

As in the proof of Theorem A, Theorem B follows readily from Corollaries 3.2 and 3.4 .

\section{REFERENCES}

[1] D. R. Adams, Weighted capacity and the Choquet integral, Proc. Amer. Math. Soc. 102 (1988), 879-887. MR934860 (89h:31007)

[2] D. R. Adams and L. I. Hedberg, Function spaces and potential theory, Springer-Verlag, Berlin-Heidelberg-New York, 1996. MR1411441 (97j:46024) 
[3] D. R. Adams and R. Hurri-Syrjänen, Vanishing exponential integrability for functions whose gradients belong to $L^{n}(\log (e+L))^{\alpha}$, J. Funct. Anal. 197 (2003), 162-178. MR.1957679 (2004b:46035)

[4] D. R. Adams and R. Hurri-Syrjänen, Capacity estimates, Proc. Amer. Math. Soc. 131 (2003), 1159-1167. MR 1948107 (2003k:46034)

[5] N. Aissaoui and A. Benkirane, Capacités dans les espaces d'Orlicz, Ann. Sci. Math. Québec 18 (1994), 1-23. MR.1273865 (95h:31009)

[6] C. Bennett and R. Sharpley, Interpolation of Operators, Academic Press, Inc., New York, 1988. MR928802(89e:46001)

[7] D. E. Edmunds and W. D. Evans, Hardy operators, function spaces and embeddings, Springer Monographs in Mathematics, Springer-Verlag, Berlin, 2004. MR2091115 (2005g:46068)

[8] T. Futamura, Y. Mizuta, T. Ohno and T. Shimomura, Orlicz-Sobolev capacity of balls, to appear in Illinois J. Math.

[9] J. Heinonen, T. Kilpeläinen and O. Martio, Nonlinear potential theory of degenerate elliptic equations, Clarendon Press, 1993. MR,1207810 (94e:31003)

[10] J. Joensuu, On null sets of Sobolev-Orlicz capacities, Illinois J. Math. 52 (2008), 1195-1211. MR2595762

[11] J. Joensuu, Orlicz-Sobolev capacities and their null sets, Rev. Mat. Complut. 23 (2010), 217-232. MR2578579

[12] J. Joensuu, Estimates for certain Orlicz-Sobolev capacities of an Euclidean ball, Nonlinear Anal. 72 (2010), 4316-4330.

[13] T. Kilpeläinen, Weighted Sobolev spaces and capacity, Ann. Acad. Sci. Fenn. Ser. A. I. Math. 19 (1994), 95-113. MR1246890 (95h:46054)

[14] S. E. Kuznetsov, Removable singularities for $L u=\Psi(u)$ and Orlicz capacities, J. Funct. Anal. 170 (2000), 428-449. MR:1740658(2001g:35078)

[15] N. G. Meyers, A theory of capacities for potentials in Lebesgue classes, Math. Scand. 8 (1970), 255-292. MR0277741 (43:3474)

[16] Y. Mizuta, Continuity properties of potentials and Beppo-Levi-Deny functions, Hiroshima Math. J. 23, 79-153 (1993). MR.1211771 (94d:31005)

[17] Y. Mizuta, Potential theory in Euclidean spaces, Gakkōtosyo, Tokyo, 1996. MR1428685 (98e:31001)

[18] Y. Mizuta, E. Nakai, T. Ohno and T. Shimomura, Sobolev embeddings for Riesz potentials of functions in Morrey spaces $L^{(1, \varphi)}(G)$, to appear in J. Math. Soc. Japan.

[19] Y. Mizuta and T. Shimomura, Differentiability and Hölder continuity of Riesz potentials of functions in Orlicz classes, Analysis 20 (2000), 201-223. MR.1778254 (2001h:46052)

[20] Y. Mizuta and T. Shimomura, Vanishing exponential integrability for Riesz potentials of functions in Orlicz classes, Illinois J. Math. 51 (2007), 1039-1060. MR2417414|(2009d:31014)

[21] Y. Mizuta and T. Shimomura, Continuity properties of Riesz potentials of Orlicz functions, Tohoku Math. J. 61 (2009), 225-240. MR2541407

[22] W. P. Ziemer, Weakly differentiable functions, Springer-Verlag, New York, 1989. MR.1014685 (91e:46046)

Department of Mathematics, Graduate School of Science, Hiroshima University, HigASHI-HiRoshima 739-8521, JAPAN

E-mail address: yomizuta@hiroshima-u.ac.jp

General Arts, Hiroshima National College of Maritime Technology, Higashino OOSAKIKAMIJIMA TOYOTAGUn 725-0231, JAPAN

E-mail address: ohno@iroshima-cmt.ac.jp

Current address: Department of Mathematics, Faculty of Education, Oita University, Dannohara Oita 870-1192, Japan

Department of Mathematics, Graduate School of Education, Hiroshima University, Higashi-Hiroshima 739-8524, JAPAN

E-mail address: tshimo@hiroshima-u.ac.jp 